\title{
DNA methylation profiling opens a new phase in the search of targeted therapy against Ewing sarcoma
}

\author{
"In time, the new phase opened by whole sequencing and \\ epigenomic landscapes will not only provide new therapeutic \\ strategies but also will change how basic research in cancer cell \\ biology is performed."
}

First draft submitted: 26 Jan 2017; Accepted for publication: 02 Feb 2017; Published online: 23 August 2017

Keywords: DNA methylation • Ewing sarcoma • targeted therapies

Cancer arises as a result of complex imbalances in the delicate machineries that regulate genomic stability and tissue homeostasis. Once originated from our cells, tumors thrive and spread, feeding from the host resources as any other parasite. During the process, tumor cells are subjected to Darwinian evolution. Cancers accumulate variations and branch into different subclonal populations with their own specificities in terms of proliferation rate, mutation burden, immunological potential, metastatic capabilities or drug resistance. Understanding the historic complexity of tumor progression thus becomes a must in the development of successful therapeutic approaches [1].

In 2005 the United States Government launched The Cancer Genome Atlas Initiative, followed by the constitution of the International Cancer Genome Consortium [2]. Their initial aim was to characterize the distribution of mutations and major karyotypic alterations. But soon was understood that single nucleotide and copy number variations fall short to explain cancer evolution. Epigenetic alterations, the epigenome, must also be taken into account [3,4]. Thus, in 2010 the International Human Epigenome Consortium was constituted to address this issue [5]. Since then, several studies encompassing sequencing and epigenetic characterization have been published. Those studies underline the major common key events in tumor evolution within the body. As a result, the genomic (and epigenomic) landscape for the major tumor entities has already been unveiled by the consortia members or by ad-hoc cooperative research teams [5,6].

This long-term redefinition of cancer research is slowly reaching other fields, like pediatric cancer. Cancer in childhood and young adults possesses its own particular characteristics. It cannot be easily linked to environmental interference or highly stressed tissues [7]. These cancers frequently arise from well protected tissues with low physiological burden (muscle, blood, etc.). The foundations of childhood cancer (rhabdomyosarcoma, leukemia, etc.) are mostly rooted in the failure of key developmental processes to onset correctly. Furthermore, the physiology of young patients put serious constraints to the application of known effective treatments, due to the dramatic side effects they cause in young organisms [8].

Ewing sarcoma (ES) is one of these cancer entities from whom our knowledge has increased straightforwardly in the past years. Since the identification of EWS/FLI1 fusion as its major driver, great advances have been made in understanding the development of this tumor [9]. Now it is possible to clearly perform an ES diagnosis in base of the presence of EWS/FLI1 (or a few functionally identical

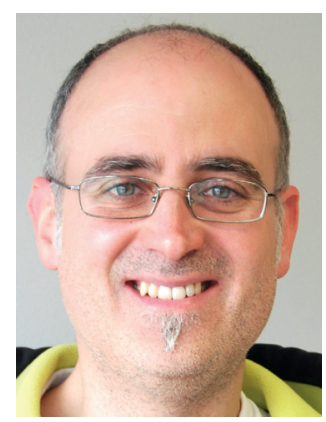

Santi Rello-Varona

Sarcoma Research Group, Institut d'Investigació Biomèdica de BellvitgeIDIBELL, L'Hospitalet de Llobregat, Barcelona 08908, Spain Tel.: +34932607429 Fax: +34932607426 srello@idibell.cat

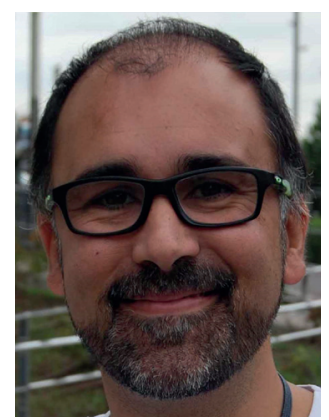

Oscar M Tirado Sarcoma Research Group, Institut d'Investigació Biomèdica de BellvitgeIDIBELL, L'Hospitalet de Llobregat, Barcelona 08908, Spain

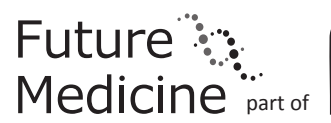


gene fusion variants). We know that EWS/FLI1 works as a transcription factor as well as a chromatin remodeler [10]. EWS/FLI1 action reprograms the cells, developing a cancerous phenotype. Silencing of EWS/FLI1 causes cell death, opening the window to yet-to-come targeted drugs.

Nevertheless, we are far away from knowing how to effectively fight this malignancy. We still lack proper models to acquire enough knowledge about the developmental cell-of-origin of ES. We need more data concerning how metastasis occurs (most of the patients have already metastatic disease at diagnosis). Furthermore, we lack effective means to early detect ES patients before symptoms (and metastasis) arises [9].

\section{"Cancer cells can present general}

hypomethylation linked to chromosomal instability and/or hypermethylation of particular $\mathrm{CpG}$ islands in the gene promoter regions (usually related to gene silencing)".

In the February issue of Cancer Letters journal, we published the first complete analysis of the DNA methylation marks in ES [11]. As a tumor entity that, apart from the EWS/FLI1 fusion, rarely shows recurrent mutations, any improvement in the knowledge of its epigenomic landscape becomes an important milestone [10]. In this sense, DNA methylation profiling provides meaningful data [4]. Cancer cells can present general hypomethylation linked to chromosomal instability and/or hypermethylation of particular $\mathrm{CpG}$ islands in the gene promoter regions (usually related to gene silencing). In the past few years, the improvement on experimental techniques and bioinformatics has permitted a boost in epigenomic characterization [12] By comparing different healthy donor samples with 15 Spaniard patient samples and 7 established cell lines, we isolated ES distinctive marks. This is important because culture cells normally diverge from the original tumors in their methylation pattern. Proper comparison provides grounds to identify those features unique to ES from those belonging to the individual patients and those resulting from the in vitro culture [13,14].

Our results show evidence of hyper-activation of certain genes, for example, CCND1 among many others. CCND1 over-expression is shared by distinct tumor entities and its epigenetic origin has also been reported in neuroblastoma [15]. In general, deregulated genes in ES can been linked generally to processes of morphogenesis and differentiation, thus concurring with the idea of ES as a developmental pathology. The complete raw data of our analysis of the ES DNA methylation landscape is freely available at the Gene Expression Omnibus website [16]. We expect the community will take advantage of it and during the following years, it will provide new potential targets against ES.

We focused our research in the PTRF gene (that codifies for the PTRF/Cavin-1 protein). PTRF is repressed in ES by hypermethylation in a putative enhancer region inside the gene and in the S-Shore close to its promoter. PTRF silencing completes the understanding of the particularly tumorigenic behavior of over-expressed CAV1 in ES [17]. Both proteins, PTRF and CAV1, are partners in the generation and function of caveolae. Caveolae are important signaling hubs configured as small flask-shaped invaginations of the plasma membrane. The presence of excesive CAV1 in mostly caveolae-less ES cells is linked to enhanced proliferative and metastatic behavior, as well as drug resistance [17]. This is consequence of CAVI being a direct target for expression by EWS/FLI1 and because PTRF is repressed through methylation. When PTRF expression is rescued, ES cells re-start to form caveolae. Caveolae restoration then leads to cell death by apoptosis in a mechanism that is $\mathrm{p} 53$ dependent via the unbalance of its regulation by MDM2.

DNA methylation profiling, whole-genome sequencing, single-cell analyses, etc. provide enormous amounts of raw data that need to be validated. High hopes have been placed in this kind of analysis. Among their large databases, we can find valuable information illustrating the role of driver mutations; or unveiling new profiles of epigenetic marks as source of particular phenotypes. With this information, scientists all over the world struggle to design new therapeutic protocols. Such approaches have been proven effective in inspiring new treatments. Indeed, they are already the source of novel targeted therapies against tumors [18]. Moreover, better knowledge of tumor molecular drivers and intratumoral heterogeneity allow to enhance stratification of patients [19]. Now, this kind of data is reaching to pediatric sarcomas and we hope that soon we can offer a knowledge leap to the design of new treatments.

Currently, US FDA approved use of epigenetic modifiers for cancer treatment, which is currently limited to two classes of agents, DNA methylation inhibitors and histone deacetylase inhibitors [20]. However, these drugs are only approved for the treatment of blood cancers. For pediatric solid tumors several Phase I clinical trials have been reported using these drugs as monotherapy, unfortunately no objective responses have been observed. Combination of these drugs with either standard chemotherapy or other targeted therapies including immunotherapy must be in the horizon of future treatment approaches in order to exploit in an intelligent way the goodness of reversing the silencing of tumor suppressor genes. Nevertheless, combination of new targeted drugs constitutes themselves into new 
selective pressures; meaning that deep knowledge of tumor evolution of resistance will be still required [21]. In fact, the data available nowadays is still insufficient to make predictions about whether tumors (and for how long) will respond to a particular treatment [21]. We know that drug resistance will eventually arise, but we are still incapable to predict the drivers of this resistance. Moreover, those analyses have also revealed how tumor cells continue to diverge after being put into in vitro culture. This allows us to clearly evaluate the representativeness and usefulness of our cell culture models. Indeed, the scientific community has to be aware of this issue. We need to be more demanding in the experimental settings and the best choosing of cell culture models. In time, the new phase opened by whole sequencing and epigenomic landscapes will

\section{References}

Papers of special note have been highlighted as: $\bullet$ of interest;

•• of considerable interest

1 Korolev KS, Xavier JB, Gore J. Turning ecology and evolution against cancer. Nat. Rev. Cancer 14(5), 371-380 (2014).

2 International Cancer Genome Consortium, Hudson TJ, Anderson W et al. International network of cancer genome projects. Nature 464(7291), 993-998 (2010).

3 Hitchins MP. Constitutional epimutation as a mechanism for cancer causality and heritability? Nat. Rev. Cancer 15(10), 625-634 (2015).

4 Heyn H, Esteller M. DNA methylation profiling in the clinic: applications and challenges. Nat. Rev. Genet. 13(10), 679-692 (2012).

5 Stunnenberg HG, Hirst M. The International Human Epigenome Consortium: a blueprint for scientific collaboration and discovery. Cell 167(5), 1145-1149 (2016).

6 Tomczak K, Czerwińska P, Wiznerowicz M. The Cancer Genome Atlas (TCGA): an immeasurable source of knowledge. Contemp. Oncol. (Pozn.) 19(1A), A68-A77 (2015).

7 Wachtel M, Schäfer BW. Targets for cancer therapy in childhood sarcomas. Cancer Treat. Rev. 36(4), 318-327 (2010).

8 Sarosiek KA, Fraser C, Muthalagu N et al. Developmental regulation of mitochondrial apoptosis by c-Myc governs ageand tissue-specific sensitivity to cancer therapeutics. Cancer Cell. 31(1), 142-156 (2016).

9 Gaspar N, Hawkins DS, Dirksen U et al. Ewing sarcoma: current management and future approaches through collaboration. J. Clin. Oncol. 33(27), 3036-3046 (2015).

10 Tomazou EM, Sheffield NC, Schmidl C et al. Epigenome mapping reveals distinct modes of gene regulation and widespread enhancer reprogramming by the oncogenic fusion protein EWS-FLI1. Cell Rep. 10(7), 1082-1095 (2015). not only provide new therapeutic strategies but also will change how basic research in cancer cell biology is performed.

\section{Financial \& competing interests disclosure}

S Rello-Varona is funded by Fundación "Alba Pérez lucha contra el cáncer infantil." OM Tirado is funded by Instituto de Salud Carlos III and European Union's Fondo Europeo de Desarrollo Regional (FEDER) "Una manera de hacer Europa/A way to achieve Europe." The authors have no other relevant affiliations or financial involvement with any organization or entity with a financial interest in or financial conflict with the subject matter or materials discussed in the manuscript apart from those disclosed.

No writing assistance was utilized in the production of this manuscript.
11 Huertas-Martínez J, Court F, Rello-Varona S et al. DNA methylation profiling identifies PTRF/Cavin-1 as a novel tumor suppressor in Ewing sarcoma when co-expressed with caveolin-1. Cancer Lett. 386, 196-207 (2017).

12 Han $Y, H e X$. Integrating epigenomics into the understanding of biomedical insight. Bioinform. Biol. Insights 10, 267-289 (2016).

13 Varley KE, Gertz J, Bowling KM et al. Dynamic DNA methylation across diverse human cell lines and tissues. Genome Res. 23(3), 555-567 (2013).

14 Hennessey PT, Ochs MF, Mydlarz WW et al. Promoter methylation in head and neck squamous cell carcinoma cell lines is significantly different than methylation in primary tumors and xenografts. PLoS ONE 6(5), e20584 (2011).

15 Gómez S, Castellano G, Mayol G et al. DNA methylation fingerprint of neuroblastoma reveals new biological and clinical insights. Epigenomics 7(7), 1137-1153 (2015).

16 NCBI. Gene Expression Omnibus. https://www.ncbi.nlm.nih.gov/geo/query/acc.

17 Sáinz-Jaspeado M, Martin-Liberal J, Lagares-Tena L, MateoLozano S, Garcia del Muro X, Tirado OM. Caveolin-1 in sarcomas: friend or foe? Oncotarget 2(4), 305-312 (2011).

18 Tatarian T, Winter JM. Genetics of pancreatic cancer and its implications on therapy. Surg. Clin. North Am. 96(6), 1207-1221 (2016).

19 Tasian SK, Hunger SP. Genomic characterization of paediatric acute lymphoblastic leukaemia: an opportunity for precision medicine therapeutics. Br. J. Haematol. 176(6), 867-882 (2016).

20 Raynal NJ-M, Da Costa EM, Lee JT et al. Repositioning FDA-approved drugs in combination with epigenetic drugs to reprogram colon cancer epigenome. Mol. Cancer Ther. (2016).

21 Lipinski KA, Barber LJ, Davies MN, Ashenden M, Sottoriva A, Gerlinger M. Cancer evolution and the limits of predictability in precision cancer medicine. Trends Cancer 2(1), 49-63 (2016). 